\title{
Maternal DDT Exposures in Relation to Fetal and 5-Year Growth
}

\author{
Todd A. Jusko*, Thomas D. KoepselI ${ }^{\star}$, Rebecca J. Baker ${ }^{\dagger}$, Teri A. Greenfield ${ }^{\ddagger}$, Eric J. \\ Willman§, M. Judith Charles§, Stuart W. Teplinף, Harvey Checkoway ${ }^{\star}, \|$, and Irva Hertz- \\ Picciotto $\ddagger$ \\ "Department of Epidemiology, School of Public Health and Community Medicine, University of \\ Washington, Seattle, WA
}

tDepartment of Epidemiology, School of Public Health, University of North Carolina-Chapel Hill, Chapel Hill, NC

¥Division of Epidemiology, Department of Public Health Sciences, School of Medicine, University of California-Davis, Davis, CA

§Department of Environmental Toxicology, University of California-Davis, Davis, CA

"Center for the Study of Development and Learning, Department of Pediatrics, University of North Carolina-Chapel Hill School of Medicine, Chapel Hill, NC

IDepartment of Environmental and Occupational Health Sciences, School of Public Health and Community Medicine, University of Washington, Seattle, WA

\begin{abstract}
Background-Dichlorodiphenyltrichloroethane (DDT) is an organo-chlorine pesticide still used in areas of the world where malaria vector control is needed. Few studies have examined in utero exposures to DDT in relation to fetal and early childhood growth in populations with substantial exposure to DDT. Furthermore, only a portion of these studies have investigated in utero exposures and growth during childhood.

Methods-To assess the role of in utero exposures to DDT on fetal and early childhood growth, we analyzed data from mothers and children who participated in the Child Health and Development Study (CHDS), a cohort study of 20,754 women and their pregnancies conducted in the San Francisco Bay area during the 1960s. We measured $p, p^{\prime}$-DDE, $o, p^{\prime}$-DDT, and $p, p^{\prime}$-DDT concentrations from the stored sera of 399 women collected during pregnancy. Outcomes were measured at the child's birth and at 5 years of age.
\end{abstract}

Results-Maternal $p, p^{\prime}$-DDE concentrations were considerable in this study, averaging 6.9 micrograms per gram lipid. After covariate adjustment, a small increase in gestational age was observed with increases in $p, p^{\prime}$-DDT and $o, p^{\prime}$-DDT, but there was no association with $p, p^{\prime}$-DDE. At 5 years of age, an increase from the 25th to the 75th percentile in $p, p^{\prime}$-DDE was related to a 2$\mathrm{mm}$ increase in head circumference ( $95 \%$ confidence interval $=0$ to 4$)$. Overall effect sizes were

Copyright $@ 2006$ by Lippincott Williams \& Wilkins.

Correspondence: Todd A. Jusko, University of Washington, Box 357236, Seattle WA 98195. jusko@u.washington.edu. Dr. Willman is currently affiliated with Ecolab, Eagen, MN 
small and imprecise. Furthermore, there was little evidence of specificity for a given outcome or exposure at either age.

Conclusions-At the concentrations studied in this sample, DDT compounds did not appear to impair fetal or 5-year growth.

Dichlorodiphenyltrichloroethane (DDT) was used widely in the United States as a pesticide until its ban in 1973. Its use was also restricted by most industrialized countries in the 1970s; however, use continues in countries where malaria vector control is still needed. Technicalgrade DDT is composed almost entirely of 2 isomers, $p, p^{\prime}$-DDT and $o, p^{\prime}$-DDT. ${ }^{1}$

Dichlorodiphenyldichloroethene (DDE) isomers $p, p^{\prime}$-DDE and $o, p^{\prime}$-DDE result from DDT that has either degraded in the environment or has been metabolized. Because of their chemical stability and water insolubility, some DDT compounds persist in the environment years after their application, bioaccumulating through the food chain. ${ }^{2}$ As a result, humans are generally exposed to DDT compounds through consumption of meats, poultry, dairy products, and fish, and during breast-feeding as infants. ${ }^{3-5}$ Moreover, studies have demonstrated that maternal concentrations of DDT are transmitted to the developing fetus through the placenta ${ }^{6}$ and that blood concentrations of DDT in infants are associated with maternal levels during pregnancy. ${ }^{7}$

Animal studies have provided evidence of specific effects and mechanisms for the action of DDT. ${ }^{1} p, p^{\prime}$-DDE and $p, p^{\prime}$-DDT have been demonstrated to inhibit androgen binding to the androgen receptor, whereas $o, p^{\prime}$-DDT binds to the estrogen receptor and induces estrogenic effects. ${ }^{8}$ Studies of pregnant rabbits given $p, p^{\prime}$-DDT during early gestation show increases in premature delivery ${ }^{9}$ and lower birth weights among offspring. ${ }^{10}$ Furthermore, evidence from observational studies of wildlife shows an association between increased DDT concentrations and premature births among California sea lions. ${ }^{11}$

Despite some data from experimental and wildlife studies, relatively few cohort studies in humans have examined the potential effects of DDT on fetal growth. ${ }^{12}$ Among 2380 American infants born between 1959 and 1966, higher concentrations of $p, p^{\prime}$-DDE in maternal serum were associated with an increased risk of preterm delivery as well as with small-for-gestational-age birth. ${ }^{13}$ Results from other, later-born cohorts have not demonstrated associations between DDT and fetal growth, perhaps owing to the sharp decline in human DDT levels since the $1970 \mathrm{~s}^{14}$ as suggested by Gladen and colleagues. ${ }^{15}$ Additionally, results from case-control studies of preterm birth and fetal growth are mixed, although these studies were limited in size and some did not account for potential confounding factors. ${ }^{16-22}$ Furthermore, previous studies of DDT exposures and postnatal growth have focused on growth during infancy ${ }^{23}$ or puberty ${ }^{24,25}$ but have generally not considered growth during early childhood. One study of early childhood growth ${ }^{26}$ relied on DDT concentrations measured at 8 years of age, rather than at birth, and thus exposure to DDT compounds was measured subsequent to early childhood growth.

The current study was designed to examine fetal and 5-year growth in relation to prenatal DDT exposure. Pregnancies in this study occurred during a time of peak DDT use in the United States, ${ }^{1}$ making this population ideal for studying the potential effects of in utero exposure on fetal and early childhood growth. 


\section{METHODS}

\section{Study Participants}

This investigation concerns a subsample of mother-child pairs from the Child Health and Development Study (CHDS), a large prospective cohort study of women who were pregnant during the 1960s and their children. ${ }^{27,28}$ Subjects had been recruited through the Kaiser Foundation Health Plan, a prepaid health system that provides comprehensive medical care to members throughout California. Members of the Kaiser Health Plan residing in the East Bay of the San Francisco Bay Area were enrolled in the study when they first contacted participating Kaiser facilities regarding a confirmed or possible pregnancy. Only those women who completed their study interview, laboratory work, and sought prenatal care at participating Kaiser facilities were included in the study. ${ }^{27}$ In total, 20,754 pregnancies were enrolled, resulting in 19,044 live births. At the time of the 5-year follow up, 17,045 motherchild pairs were still under observation.

To select participants for the current study, we first restricted the total CHDS sample to the 3412 children who were selected for and underwent an extensive physical examination at 5 years of age. By CHDS design, these children had to have resided in the San Francisco Bay area at the time of the 5-year assessment and had to have been born between April 1964 and April 1967.

From the 3412 children assessed at 5 years of age, we excluded women who were unmarried minors at the time of their child's birth or whose enrollment interview was incomplete or completed after delivery $(\mathrm{n}=587)$. Next, we excluded pregnancies from which a second- or third-trimester blood specimen was not drawn or an insufficient volume was available ( $\mathrm{n}=$ 177). Pregnancies with multiple births were also excluded $(n=28)$. Because we were also interested in examining cognitive outcomes and potential hypothyroid effects in this sample, we further excluded the following groups: children with severe abnormalities and those who did not complete 2 cognitive examinations, a hearing examination, and a speech/language assessment $(\mathrm{n}=227)$; mothers who were deaf, had rubella during pregnancy, were taking thyroid medication in the 60 days before blood draw, or who took iodine-containing medication during pregnancy or in the 6 months before conception $(n=141)$; and infants born earlier than 35 weeks or greater than 45 weeks of gestation or for whom gestational age was unknown $(n=99)$. When mothers $(n=57)$ had more than one eligible child, no more than one was randomly selected. We then selected participants residing in a limited group of counties to enable a more complete follow-up of the adults, resulting in the exclusion of 805 children.

After these exclusions, there remained 1291 eligible children. From these, we sampled all children whose standardized score on the Raven's Progressive Matrices or Pea-body Picture Vocabulary Test was below the 10th percentile or who failed a hearing examination (stratum $\mathrm{n}=304$ ). From the remaining 987 children who scored above the 10th percentile on both examinations and passed the hearing screening, approximately $17 \%$ were randomly chosen (stratum $n=175$ ). The current sample is thus a pooled sample of these 2 strata. This sampling procedure was done to enhance the power for the hypotheses related to neurobehavioral deficits ${ }^{29}$ (which were of relatively low prevalence) while not adversely 
affecting efficiency for other hypotheses, ${ }^{30}$ including those related to growth, the subject of this article. Of the 479 mother-child pairs selected, serum DDT and lipid concentrations were determined for 399 of the mothers. Major reasons DDT and lipid concentrations could not be determined were insufficient volume, breakage, and laboratory error.

\section{Measurement and Analysis of Blood Samples}

Approximately $30 \mathrm{~mL}$ of blood was drawn from women during pregnancy. Samples were then centrifuged and the sera aliquoted and stored at $-20^{\circ} \mathrm{C}$ at National Institutes of Health (NIH) facilities. A subset (those born 1 April 1966 or later) were stored locally and then shipped to NIH. Blood draws selected for this study occurred between 3.4 and 9.3 months gestation for all women. The 10th, 25th, 50th, 75th, and 90th percentiles for gestational age of blood draws were: 4.1, 4.9, 5.6, 6.2, and 7.4 months, respectively.

For the current study, specimens were thawed, aliquoted, frozen, and sent to the University of California, Davis on dry ice for organochlorine determination. From the original serum samples, $1.00 \mathrm{~mL}$ was made available for the lipid and organochlorine analyses in the current study. Briefly, serum specimens were analyzed by gas chromatography (HewlettPackard, Palo Alto, CA) with electron capture detection using an RTX-5MS and an RTX-1701 column. ${ }^{31,32}$ Concentrations of $o, p^{\prime}$-DDE, $p, p^{\prime}$-DDE, $o, p^{\prime}$-DDT, and $p, p^{\prime}$-DDT were determined. All samples were analyzed between 14 January 1998 and 22 September 1999 in batches of 10 to 20 samples on 25 different days. Concentrations were adjusted for percent recovery for each sample. Specimens analyzed on batch dates with extremely high or low variability were reanalyzed and the 2 values averaged. Within-batch coefficients of variation (CVs) were calculated for each measure of DDT. For comparability with another study that used specimens from the CHDS, ${ }^{33}$ we first calculated the CV using the standard formula in which the standard deviation of each replicate pair was divided by the mean of each replicate pair and the median value across all replicate pairs was taken as an overall measure of precision. Calculated this way, the CV was $5.6 \%$ for $p, p^{\prime}$-DDE, $5.0 \%$ for $p, p^{\prime}-$ DDT, and $11.1 \%$ for $o, p^{\prime}$-DDT. However, when the standard deviation of each replicate is not independent of its mean, Rosner ${ }^{34}$ suggests log-transforming all values and estimating the within-subject mean square through a one-way random-effects analysis of variance. The square root of this value gives the $\mathrm{CV}$ on the original, untransformed scale. Calculated in this way, the CV was $26.9 \%$ for $p, p^{\prime}$-DDT, $22.6 \%$ for $p, p^{\prime}$-DDT, and $36.7 \%$ for $o, p^{\prime}$-DDT. Values below the limit of quantification (LOQ) (one value for $p, p^{\prime}$-DDE and 8 values for $o, p$ '-DDT) were imputed as described previously. ${ }^{32} o, p^{\prime}$-DDE coeluted during analysis and could not be quantified. ${ }^{32}$ Consequently, total DDT ( $\Sigma$ DDT) was calculated by summing $p, p^{\prime}$-DDE, $p, p^{\prime}$-DDT, and $o, p^{\prime}$-DDT.

Additionally, lipid levels were measured to allow estimation of organochlorines on a perlipid basis. Triglycerides and total cholesterol were measured using standard enzymatic techniques. ${ }^{35-37}$ Total lipid levels were estimated using the following formula: total lipids = $(2.27 \times$ total cholesterol $)+$ triglyceride $+0.623 .^{38}$ 


\section{Fetal Growth}

Fetal outcomes included birth weight, gestational age, birth length, head circumference, and gestational age-adjusted birth weight. Gestational age at delivery was calculated by subtracting the date of the last menstrual period reported by the mother from the date of delivery in the medical chart. If month of last menstrual period was known but day was not, maternal reports of the "beginning of the month" were coded as the 7th of the month, the "end of the month" as the 23rd, and no day information as the 15th of the month. Adjusted birth weight was expressed as a z-score representing the deviation from mean birth weights among all U.S. births in 1989 at each week of gestation, stratified by sex, race, and parity. ${ }^{39}$

\section{Five-Year Growth}

At 5 years of age, a pediatrician conducted an extensive physical examination, which included anthropometric measures of weight, height, sitting height, biacromial distance (shoulder breadth), chest breadth (maximum breadth across the rib cage), chest depth (maximum horizontal depth of the chest at the level of the nipples), and bi-iliac distance (maximum breadth across the iliac crests of the pelvis). Race- and sex-standardized measures of weight and height were also recorded and are presented as a z-score. Weight was measured in pounds and ounces, and height and sitting height were measured to the 16th of an inch. Biacromial distance, chest breadth, chest depth, and bi-iliac distance were measured in millimeters with the Harpenden digital anthropometer. ${ }^{27}$

\section{Covariates}

Mothers were interviewed during pregnancy to collect information about medical and reproductive history, demographic information about the gravida and her family, and information about smoking and drinking habits. ${ }^{27}$ Paternal information was also collected. Additionally, medical records were abstracted to determine medication use during pregnancy, previous and chronic illnesses, and whether or not the child was breastfed. Medications used during pregnancy were reviewed to determine their potential to affect intrauterine growth. To do this, current references and those appropriate to the time period of the study were consulted to ascertain each drug's formulation and active ingredients. ${ }^{40-44}$ These formulations and ingredients were then checked against current references with regard to effects on intrauterine growth, and mothers were coded yes/no for medications prescribed or taken during pregnancy that have been reported to have an association with intrauterine growth. Twelve medications were identified as potentially disruptive to intrauterine growth. These medications fell into 4 general categories: tetracycline and derivatives, analgesics, barbiturates, and amphetamines/central nervous system stimulants. Henceforth these are referred to as intrauterine growth restriction (IUGR) medications.

From this information, potential covariates for our statistical models were selected based on known predictors of fetal and early childhood growth. Maternal factors considered as potential covariates were: age, race/ethnicity, education, occupation, height, prepregnancy body mass index (BMI), parity, adequacy of prenatal care, ${ }^{45}$ smoking and alcohol consumption during pregnancy, IUGR medications used during pregnancy, chronic hypertension, preeclampsia, and diabetes. Paternal height, occupation, and education were also considered as potential covariates as was child's sex. 


\section{Data Analysis}

Multiple linear regression was used to estimate the association between each measure of DDT exposure ( $p, p^{\prime}$-DDE, $p, p^{\prime}$-DDT, $o, p^{\prime}$-DDT, and $\Sigma$ DDT) and each outcome where DDT exposure was modeled continuously as a lipid-adjusted value (in micrograms per gram of lipid). We developed two sets of covariates, one for the outcomes measured at delivery, and the other for outcomes at 5 years of age. For each set, we first identified all variables associated at the $P<0.20$ level with any outcome at that age regardless of each variable's ultimate association with exposure. Then, to identify the most important confounding variables, $\Sigma$ DDT was added to a regression model for each outcome and each covariate tested to determine whether its exclusion changed the $\Sigma$ DDT regression coefficient by more than $10 \%$. If a variable was determined to be a confounder by this criterion, it was included in all models for that age regardless of the dependent variable. Additionally, all models were adjusted for the duration of pregnancy at time of blood draw and 2 specimen characteristics: batch-date of laboratory analysis and an indicator of storage history (only at an NIH facility or stored on-site and later shipped to the facility). ${ }^{32}$ Three women with diabetes were excluded from all fetal growth models. To account for potentially greater homogeneity within sampling strata, we used the SURVEYREG procedure in SAS (version 9.1; SAS Institute, Inc., Cary, NC) with design effects to account for the stratified sampling without replacement; we also applied weights to ensure that our estimates were generalizable to all children in the CHDS who met the current study's eligibility criteria. Weights were proportional to the inverse of the sampling fractions. Sampling weights were 1.27 for the children whose standardized score on the Raven's Progressive Matrices or Peabody Picture Vocabulary Test was below the 10th percentile or who failed a hearing examination and 6.21 for the remaining children who scored above the 10th percentile on both examinations and passed the hearing test.

Based on previous findings from the literature ${ }^{26,46}$ and the potential estrogenic effect of $o, p$ '-DDT and the antian-drogenic effects of $p, p^{\prime}$-DDE and $p, p^{\prime}$-DDT, we also examined effect modification by child's sex by adding a sex*exposure term to each model. A variable representing the sum of maternal lipid-adjusted polychlorinated biphenyl (PCB) congeners (IUPAC \#105, \#110, \#118, \#137, \#138, \#153, \#170, \#180, and \#187) was also added to each regression model to determine whether PCB exposure confounded the association between DDT and growth. ${ }^{47}$

\section{RESULTS}

\section{Sample Characteristics}

Table 1 compares the distributions of maternal, paternal, and child characteristics for subjects in each sampling stratum and those included in our final sample to those participating in the original CHDS population. Differences in the distributions of most of the variables listed in Table 1 can be seen when comparing the 2 sampling strata, thus motivating the use of weighted regression techniques. Maternal age, parity, prepregnancy BMI, occupation, education, prenatal care, and smoking status were similar among mothers in the current study to those participating in the CHDS cohort. Mothers in the current study were more likely to be black, born in the southeast United States, less likely to consume at 
least one drink per week during pregnancy, less likely to be prescribed medications that affected intrauterine growth, and slightly more likely to be diagnosed with preeclampsia, eclampsia, or essential hypertension. Because black race and birth in the Southeast were also strongly associated with DDT concentration in this sample, ${ }^{32}$ women participating in the current study are likely to have higher levels of DDT than mothers in the CHDS not included in our sample. Compared with the CHDS cohort, fathers in the current study were less likely to be employed in professional or technical positions or as students and were less likely to be college graduates.

The mean and median values along with the 25th and 75th percentile of each lipid-adjusted DDT measure are given in Table 2. As expected, maternal serum concentrations of $p, p^{\prime}-$ DDE (the main metabolite of $p, p^{\prime}$-DDT) were the highest with a mean approximately 3.5 times that of $p, p^{\prime}$-DDT. The distributions of fetal and 5-year growth outcomes are also given in Table 2.

\section{Fetal Growth}

Before adjustment for potential confounders, we observed lower birth weight to be associated with several of the measures of DDT exposure; the strongest of these was with $\Sigma$ DDT(a 58-g reduction in birth weight comparing the 75th with the 25th percentile of $\Sigma$ DDT; 95\% confidence interval $[\mathrm{CI}]=-120$ to 3 ). However, none of these associations with birth weight were statistically significant (data not shown).

Table 3 presents fetal growth results after adjusting for maternal BMI, height, parity, race, and alcohol intake as well as father's education and occupation. Overall, there was little association observed between measures of DDT and fetal growth. Results were inconsistent with regard to specific exposures or outcomes and effect sizes were small and sometimes had wide confidence limits. Increases in gestational age were observed for increasing maternal concentrations of $p, p^{\prime}$-DDT and $o, p^{\prime}$-DDT, although this association was weak. Comparing the 75th to the 25th percentile, there was a 1.7-day increase in gestation length with $p, p^{\prime}$-DDT concentration and a 1.8-day increase with $o, p^{\prime}$-DDT concentration. Some heterogeneity by sex was observed for birth weight across all measures of DDT exposure with males appearing to be more affected; however, the difference was small (data not shown). Additional adjustment for PCB concentration did not substantially change the results given in Table 3 .

\section{Five-Year Growth}

Overall, few noteworthy associations were seen between the 4 measures of DDT and the 9 measures of growth at 5 years of age in either adjusted or unadjusted models. Results for height, weight, and head circumference measures are given in Table 4. After adjusting for maternal BMI, height, race, and quality of prenatal care (inadequate, adequate, intermediate, or more than adequate ${ }^{45}$ ), head circumference was $2 \mathrm{~mm}$ greater among children at the 75 th percentile of $p, p^{\prime}$-DDE concentration compared with children at the 25 th percentile (95\% CI $=0$ to 4). A similar association was also observed for $\Sigma$ DDT. Estimates for biacromial distance, chest breadth and depth, and bi-iliac distance were small and imprecise and showed no important associations with any of the measures of DDT (data not shown). No 
consistent or noteworthy heterogeneity by child's sex was observed in relation to DDT exposure and 5-year growth. Similar to the fetal growth outcomes, additional adjustment for PCB concentration did not alter our findings.

\section{DISCUSSION}

We found little evidence of associations between in utero DDT exposure in the ranges of exposure studied and fetal and early childhood growth. Despite adjustment for maternal and paternal factors, estimated effect sizes were small and imprecise, and there were no consistent patterns of association across exposures or outcomes.

Four other major cohort studies ${ }^{13,15,33,48}$ have examined the association between in utero DDT exposure and fetal growth with equivocal results. In 2 of these studies, ${ }^{15,48}$ DDT concentrations were considerably lower than those in the current study and both found little evidence of effects on fetal growth. In one of these, Rogan and colleagues ${ }^{48}$ measured maternal body burden of $p, p^{\prime}$-DDE in a cohort of 912 children born between 1978 and 1982 in North Carolina; like the current study, they found no association between $p, p^{\prime}$-DDE levels and either birth weight or head circumference. In another study with lower maternal DDT exposures than the current study, Gladen and colleagues ${ }^{15}$ measured $p, p^{\prime}$-DDE and $p, p^{\prime}-$ DDT concentrations in 197 mothers giving birth between 1993 and 1994 to singleton infants in the Ukraine. Although unadjusted models and those adjusted for gestational age showed increasing birth weight with increasing maternal concentrations of $p, p^{\prime}$-DDE, this association disappeared after adjustment for other potential confounding factors such as maternal age, height, and parity.

The other 2 cohort studies of note included mothers with DDT concentrations that were similar to those in the current study. ${ }^{13,33}$ In a study of 2,380 children from the Collaborative Perinatal Project, born between 1959 and 1966 in the United States, increasing maternal $p, p$ '-DDE concentrations were associated with an increased risk of preterm and small-forgestational-age birth. ${ }^{13}$ Specifically, the risk of pre-term birth was approximately 3 times greater among mothers with $p, p^{\prime}$-DDE serum concentrations of at least $60 \mu \mathrm{g} / \mathrm{L}$ compared with mothers who had concentrations below $15 \mu \mathrm{g} / \mathrm{L}$ contrary to our results of increased gestational age with increased concentrations of $p, p^{\prime}$-DDT and $o, p^{\prime}$-DDT. However, a limitation of our study is that we selected infants who completed at least 35 weeks of gestation. Therefore, our gestational age results are not directly comparable with those from the Collaborative Perinatal Project. ${ }^{13}$

A study by Farhang and colleagues ${ }^{33}$ also selected mothers and infants from the same San Francisco cohort on which we based our study. Their participants had been selected from an earlier case-control study of male genital abnormalities within the cohort ${ }^{49}$ and so their sample included only male infants. The authors noted that increases in maternal $p, p^{\prime}$-DDE and $p, p^{\prime}$-DDT reduced the risk of small-for-gestational-age birth, although the estimates were imprecise. We observed similar associations for $p, p^{\prime}$-DDT and $o, p^{\prime}$-DDT in relation to gestational age at birth, although we had excluded infants less than 35 weeks gestation. Overall, however, the study by Farhang and colleagues ${ }^{33}$ found little association between measures of DDT and fetal growth as we observed in our data sampled from the same 
population. Some authors ${ }^{15}$ have suggested that differences in the magnitude of maternal exposure may explain positive and negative study results with only high concentrations of DDT exposure lowering birth weight. Our findings and those of Farhang and colleagues, however, do not support this assertion, at least not at the exposure levels observed.

With regard to postnatal effects, few studies have examined DDT exposure in relation to growth beyond infancy, and most have focused on anthropometric measures at puberty, ${ }^{24,25}$ making it difficult to compare our 5-year growth results with previous study findings. In the North Carolina cohort, height and height-adjusted weight at puberty among boys showed a positive association with transplacental exposures to $p, p^{\prime}$-DDE. ${ }^{25}$ In the Collaborative Perinatal Project, ${ }^{24}$ none of the prenatal measures of DDT compounds were associated with height, ratio of sitting height to height, BMI, and skeletal age at puberty. We observed little association between DDT exposures and height and weight at 5 years. Karmaus and colleagues $^{26}$ studied 343 German children to determine whether DDE concentrations measured at 8 years of age were predictive of children's growth from birth through 10 years of age. In that study, growth in girls was inversely associated with exposure to DDE. This association between DDE and growth was not observed when girls were examined at 10 years of age, however. Additionally, no association between DDE and growth was observed among boys. However, any of these findings might well be biased, in an unknown direction, by the use of an exposure measurement obtained at 8 years of age. In contrast, 5-year growth was not associated with prenatal exposures to DDT in our study, and sex-specific associations were not observed.

Several potential limitations of our study should be noted, particularly when interpreting our null results. Data on postnatal exposures to DDT were not available, and thus we were unable to assess the potential effects of both pre- and postnatal exposure on 5-year growth. Information on breast-feeding was not systematically collected. However, few women breastfed their infants in those years, limiting our ability to draw conclusions about potential exposures from this source. To the extent that postnatal exposures in children were different from prenatal exposures or acted differently on growth, our results at 5 years of age may have been biased toward the null. On the other hand, the lack of multiple measures of in utero exposure is unlikely to be a concern in terms of misclassification of maternal exposure as a study of stored serum samples from the Collaborative Perinatal Project indicated that $p, p^{\prime}$-DDE concentrations were highly stable throughout pregnancy with correlation coefficients of at least 0.82 between any 2 trimesters. ${ }^{50}$

Another limitation may involve our selection process, because we selected only women whose pregnancies lasted at least 35 weeks. Thus, if the association between DDT and growth is apparent only in more vulnerable preterm infants, our study could not have detected such an association. Furthermore, there is some evidence that increasing maternal DDT concentrations increase a woman's risk of spontaneous abortion or early fetal loss. ${ }^{51-53}$ Thus, by selecting only live births, we may be missing other adverse pregnancy outcomes and are focusing attention on the healthy survivors.

The validity of our data is supported first by the confirmation of known risk factors for IUGR (most notably maternal smoking, low maternal BMI, and female sex), and, second, by 
the comparability of our organochlorine concentrations to another sample of women taking part in the CHDS $^{33,49}$ and to other historical samples from a similar time period. ${ }^{13}$

In summary, we observed little association between in utero exposures to DDT and fetal and early childhood growth. Sex-specific effects on fetal and early childhood growth were also not observed despite examining several estrogenic and antiandrogenic DDT compounds. To our knowledge, this is the first large study to examine in utero exposures to DDT in relation to growth during childhood and to assess the relation between the estrogenic DDT isomer $o, p^{\prime}$-DDT and in utero growth.

\section{Acknowledgments}

Supported by U.S. National Institutes of Health (NIH) grants R01-ES08316, P01-ES11269, and T32-ES07262 from the National Institute of Environmental Health Sciences (NIEHS), grant R01-CA96525 from the National Cancer Institute (NCI), and grants N01-HD1334 and N01-HD63258 from the National Institute of Child Health and Human Development (NICHD).

The authors thank Jean Keller for programming assistance and Bobbie Christianson for her help navigating the CHDS dataset.

\section{References}

1. Toxicological Profile for DDT, DDE, DDD. Atlanta: Agency for Toxic Substances and Disease Registry; 2002.

2. Turusov V, Rakitsky V, Tomatis L. Dichlorodiphenyltrichloroethane (DDT): ubiquity, persistence, and risks. Environ Health Perspect. 2002; 110:125-128. [PubMed: 11836138]

3. Gartrell MJ, Craun JC, Podrebarac DS, et al. Pesticides, selected elements, and other chemicals in adult total diet samples, October 1980-March 1982. J Assoc Off Anal Chem. 1986a; 69:146-159. [PubMed: 3949687]

4. Gartrell MJ, Craun JC, Podrebarac DS, et al. Pesticides, selected elements, and other chemicals in infant and toddler total diet samples, October 1980-March 1982. J Assoc Off Anal Chem. 1986b; 69:123-145. [PubMed: 3949685]

5. Jensen AA. Chemical contaminants in human milk. Residue Rev. 1983; 89:1-128. [PubMed: 6316441]

6. Cariati E, Acanfora L, Branconi F, et al. p-p'DDT in perinatal samples: report on maternal and neonatal measurements. Biol Res Pregnancy Perinatol. 1983; 4:169-171. [PubMed: 6652172]

7. O'Leary JA, Davies JE, Edmundson WF, et al. Transplacental passage of pesticides. Am J Obstet Gynecol. 1970; 107:65-68. [PubMed: 5462630]

8. Kelce WR, Stone CR, Laws SC, et al. Persistent DDT metabolite p, p'-DDE is a potent androgen receptor antagonist. Nature. 1995; 375:581-585. [PubMed: 7791873]

9. Hart MM, Adamson RH, Fabro S. Prematurity and intrauterine growth retardation induced by DDT in the rabbit. Arch Int Pharmacodyn Ther. 1971; 192:286-290. [PubMed: 5093202]

10. Fabro S, McLachlan JA, Dames NM. Chemical exposure of embryos during the preimplantation stages of pregnancy: mortality rate and intrauterine development. Am J Obstet Gynecol. 1984; 148:929-938. [PubMed: 6711631]

11. DeLong R, Gilmartin WG, Simpson JG. Premature births in California sea lions: association with high organochlorine pollutant residue levels. Science. 1973; 181:1168-1170. [PubMed: 4199282]

12. Longnecker MP, Rogan WJ, Lucier G. The human health effects of DDT (dichlorodiphenyltrichloroethane) and PCBS (polychlorinated biphenyls) and an overview of organochlorines in public health. Annu Rev Public Health. 1997; 18:211-244. [PubMed: 9143718]

13. Longnecker MP, Klebanoff MA, Zhou H, et al. Association between maternal serum concentration of the DDT metabolite DDE and preterm and small-for-gestational-age babies at birth. Lancet. 2001; 358:110-114. [PubMed: 11463412] 
14. Smith D. Worldwide trends in DDT levels in human breast milk. Int J Epidemiol. 1999; 28:179_ 188. [PubMed: 10342677]

15. Gladen BC, Shkiryak-Nyzhnyk ZA, Chyslovska N, et al. Persistent organochlorine compounds and birth weight. Ann Epidemiol. 2003; 13:151-157. [PubMed: 12604157]

16. Berkowitz GS, Lapinski RH, Wolff MS. The role of DDE and polychlorinated biphenyl levels in preterm birth. Arch Environ Contam Toxicol. 1996; 30:139-141. [PubMed: 8579383]

17. O’Leary JA, Davies JE, Feldman M. Spontaneous abortion and human pesticide residues of DDT and DDE. Am J Obstet Gynecol. 1970; 108:1291-1292. [PubMed: 5482864]

18. Procianoy RS, Schvartsman S. Blood pesticide concentration in mothers and their newborn infants. Relation to prematurity. Acta Paediatr Scand. 1981; 70:925-928. [PubMed: 7324948]

19. Siddiqui MK, Srivastava S, Srivastava SP, et al. Persistent chlorinated pesticides and intra-uterine foetal growth retardation: a possible association. Int Arch Occup Environ Health. 2003; 76:75-80. [PubMed: 12592586]

20. Wassermann M, Ron M, Bercovici B, et al. Premature delivery and organochlorine compounds: polychlorinated biphenyls and some or-ganochlorine insecticides. Environ Res. 1982; 28:106-112. [PubMed: 6179776]

21. Saxena MC, Siddiqui MK, Seth TD, et al. Organochlorine pesticides in specimens from women undergoing spontaneous abortion, premature or full-term delivery. J Anal Toxicol. 1981; 5:6-9. [PubMed: 7218781]

22. Saxena MC, Siddiqui MK, Bhargava AK, et al. Role of chlorinated hydrocarbon pesticides in abortions and premature labour. Toxicology. 1980; 17:323-331. [PubMed: 7210017]

23. Rogan WJ, Gladen BC, McKinney JD, et al. Polychlorinated biphenyls (PCBs) and dichlorodiphenyl dichloroethene (DDE) in human milk: effects on growth, morbidity, and duration of lactation. Am J Public Health. 1987; 77:1294-1297. [PubMed: 3115123]

24. Gladen BC, Klebanoff MA, Hediger ML, et al. Prenatal DDT exposure in relation to anthropometric and pubertal measures in adolescent males. Environ Health Perspect. 2004; 112:1761-1767. [PubMed: 15579424]

25. Gladen BC, Ragan NB, Rogan WJ. Pubertal growth and development and prenatal and lactational exposure to polychlorinated biphenyls and dichlorodiphenyl dichloroethene. J Pediatr. 2000; 136:490-496. [PubMed: 10753247]

26. Karmaus W, Asakevich S, Indurkhya A, et al. Childhood growth and exposure to dichlorodiphenyl dichloroethene and polychlorinated biphenyls. J Pediatr. 2002; 140:33-39. [PubMed: 11815761]

27. Child Health and Development Study. Data Archive and User's Manual of the Child Health and Development Studies, Version 2.0. Vol. 1 and 2. Berkeley, CA: School of Public Health, University of California at Berkeley and Western Consortium for Public Health; 1994.

28. van den Berg BJ, Christianson RE, Oechsli FW. The California Child Health and Development Studies of the School of Public Health, University of California at Berkeley. Paediatr Perinat Epidemiol. 1988; 2:265-282. [PubMed: 3070486]

29. Hertz-Picciotto I, Greenfield T, Teplin S, et al. Prenatal exposure to heptachlor epoxide and early childhood development. Neurotoxicology. 2004; 25:701.

30. Lee AJ, McMurchy L, Scott AJ. Re-using data from case-control studies. Stat Med. 1997; 16:1377-1389. [PubMed: 9232759]

31. Willman EJ, Hertz-Picciotto I, Keller JA, et al. A reproducible approach to the reporting of organochlorine compounds in epidemiologic studies. Chemosphere. 2001; 44:1395-1402. [PubMed: 11513118]

32. James RA, Hertz-Picciotto I, Willman E, et al. Determinants of serum polychlorinated biphenyls and organochlorine pesticides measured in women from the child health and development study cohort, 1963-1967. Environ Health Perspect. 2002; 110:617-624. [PubMed: 12117636]

33. Farhang L, Weintraub JM, Petreas M, et al. Association of DDT and DDE with birth weight and length of gestation in the Child Health and Development Studies, 1959-1967. Am J Epidemiol. 2005; 162:717-725. [PubMed: 16120698]

34. Rosner, B. Fundamentals of Biostatistics. 5. Pacific Grove, CA: Duxbury Press; 2000.

35. Wiebe DA, Bernert JT Jr. Influence of incomplete cholesteryl ester hydrolysis on enzymic measurements of cholesterol. Clin Chem. 1984; 30:352-356. [PubMed: 6697478] 
36. Kohlmeier M. Direct enzymic measurement of glycerides in serum and in lipoprotein fractions. Clin Chem. 1986; 32:63-66. [PubMed: 3510098]

37. Siedel J, Hagele EO, Ziegenhorn J, et al. Reagent for the enzymatic determination of serum total cholesterol with improved lipolytic efficiency. Clin Chem. 1983; 29:1075-1080. [PubMed: 6851096]

38. Phillips DL, Pirkle JL, Burse VW, et al. Chlorinated hydrocarbon levels in human serum: effects of fasting and feeding. Arch Environ Contam Toxicol. 1989; 18:495-500. [PubMed: 2505694]

39. Zhang J, Bowes WA Jr. Birth-weight-for-gestational-age patterns by race, sex, and parity in the United States population. Obstet Gynecol. 1995; 86:200-208. [PubMed: 7617350]

40. Physicians' Desk Reference to Pharmaceutical Specialties and Biologicals. 16. Oradell, NJ: Medical Economics; 1962.

41. Physicians' Desk Reference to Pharmaceutical Specialties and Biologicals. 19. Oradell, NJ: Medical Economics; 1965.

42. Physicians' Desk Reference to Pharmaceutical Specialties and Biologicals. 22. Oradell, NJ: Medical Economics; 1968.

43. Physicians' Desk Reference to Pharmaceutical Specialties and Biologicals. 54. Oradell, NJ: Medical Economics; 2000.

44. Behrman, R.; Kliegman, R.; Jenson, H., editors. Nelson Textbook of Pediatrics. 16. Philadelphia: WB Saunders Co; 2000.

45. Kotelchuck M. An evaluation of the Kessner Adequacy of Prenatal Care Index and a proposed Adequacy of Prenatal Care Utilization Index. Am J Public Health. 1994; 84:1414-1420. [PubMed: 8092364]

46. Hertz-Picciotto I, Charles MJ, James RA, et al. In utero polychlorinated biphenyl exposures in relation to fetal and early childhood growth. Epidemiology. 2005; 16:648-656. [PubMed: 16135941]

47. Karmaus W, Zhu X. Regarding 'Persistent organochlorine compounds and birth weight.' Ann Epidemiol. 2004; 14:151. author reply 152-153. [PubMed: 15018889]

48. Rogan WJ, Gladen BC, McKinney JD, et al. Neonatal effects of transplacental exposure to PCBs and DDE. J Pediatr. 1986; 109:335-341. [PubMed: 3090217]

49. Bhatia R, Shiau R, Petreas M, et al. Organochlorine pesticides and male genital anomalies in the child health and development studies. Environ Health Perspect. 2005; 113:220-224. [PubMed: 15687061]

50. Longnecker MP, Klebanoff MA, Gladen BC, et al. Serial levels of serum organochlorines during pregnancy and postpartum. Arch Environ Health. 1999; 54:110-114. [PubMed: 10094288]

51. Longnecker MP, Klebanoff MA, Dunson DB, et al. Maternal serum level of the DDT metabolite DDE in relation to fetal loss in previous pregnancies. Environ Res. 2005; 97:127-133. [PubMed: 15533328]

52. Korrick SA, Chen C, Damokosh AI, et al. Association of DDT with spontaneous abortion: a casecontrol study. Ann Epidemiol. 2001; 11:491-496. [PubMed: 11557181]

53. Venners SA, Korrick S, Xu X, et al. Preconception serum DDT and pregnancy loss: a prospective study using a biomarker of pregnancy. Am J Epidemiol. 2005; 162:709-716. [PubMed: 16120699]

Epidemiology. Author manuscript; available in PMC 2014 June 03. 


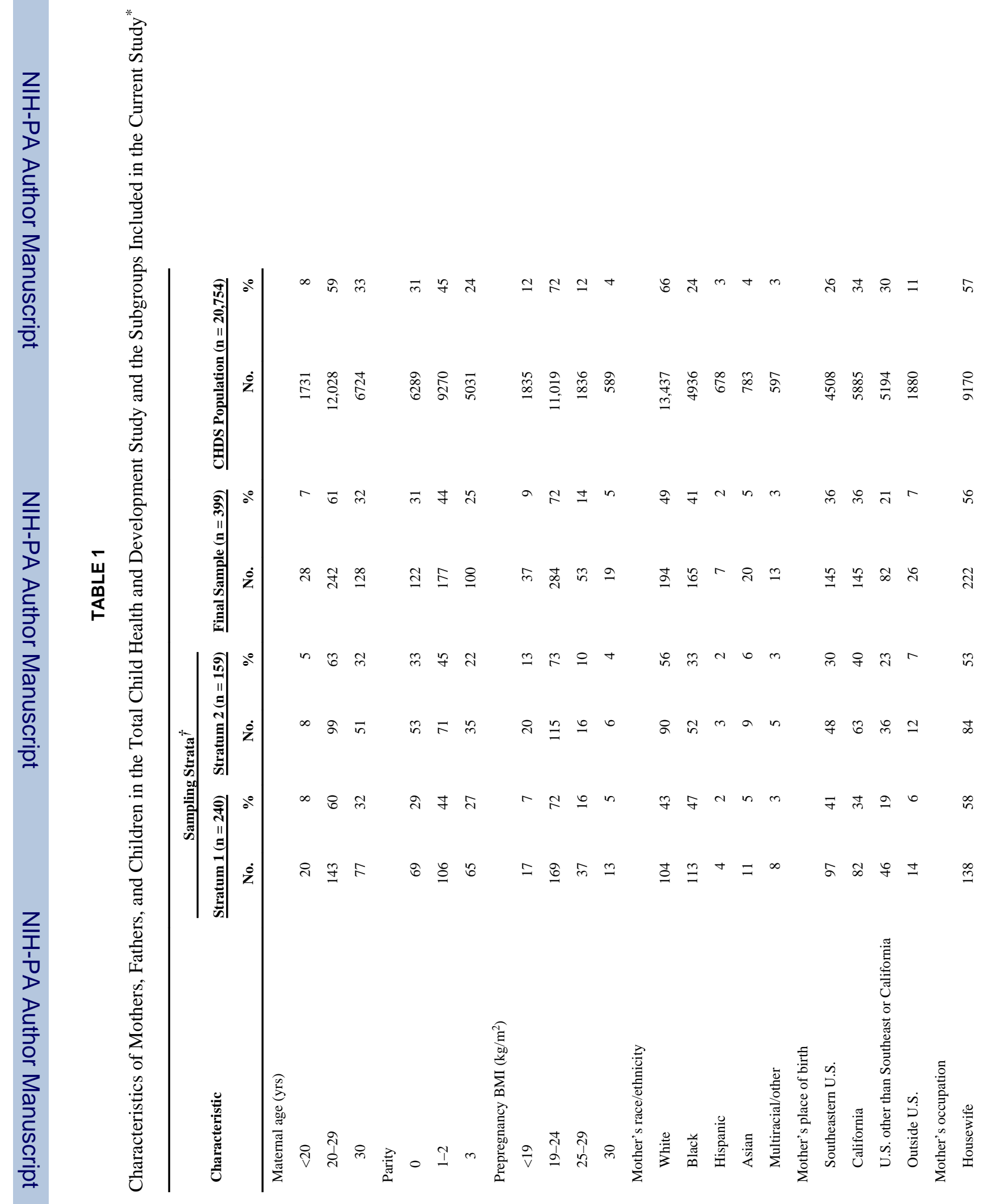




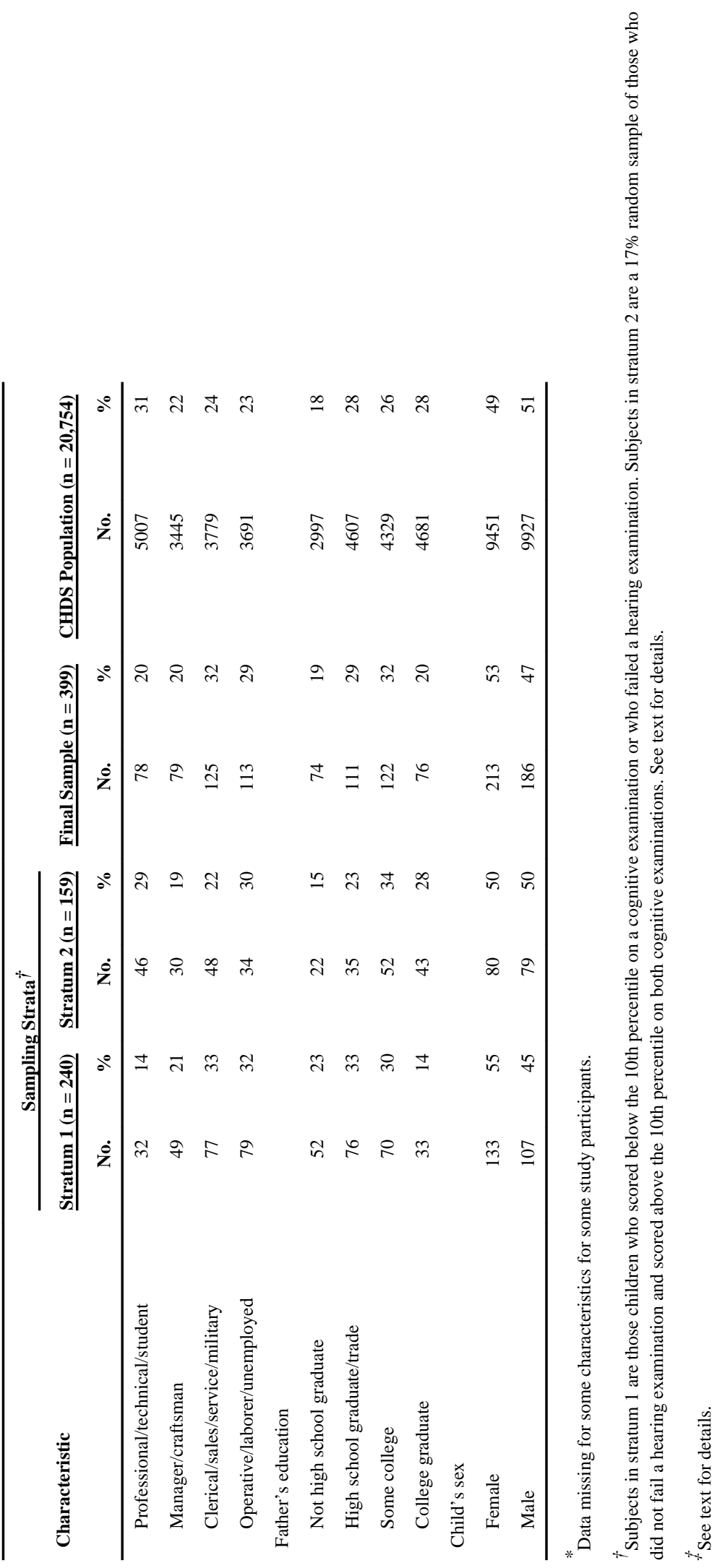

Epidemiology. Author manuscript; available in PMC 2014 June 03. 
TABLE 2

Distributions of Fetal and Early Childhood Growth Outcomes and DDT Concentrations

\begin{tabular}{|c|c|c|c|c|}
\hline Characteristic & No. & Mean \pm SD & Median & 25th, 75th Percentile \\
\hline \multicolumn{5}{|l|}{ DDT concentrations (lipid-adjusted, in $\mu \mathrm{g} / \mathrm{g}$ lipid) } \\
\hline$p, p^{\prime}-\mathrm{DDE}$ & 399 & $6.85 \pm 4.80$ & 5.88 & $3.90,8.56$ \\
\hline$p, p^{\prime}-\mathrm{DDT}$ & 399 & $1.93 \pm 1.25$ & 1.61 & $1.11,2.30$ \\
\hline$o, p^{\prime}$-DDT & 399 & $0.27 \pm 0.22$ & 0.20 & $0.12,0.35$ \\
\hline$\Sigma$ DDT & 399 & $9.05 \pm 5.60$ & 7.95 & $5.68,11.15$ \\
\hline \multicolumn{5}{|l|}{ Fetal growth outcomes } \\
\hline Birth weight (g) & 399 & $3298 \pm 448$ & 3345 & 2977,3600 \\
\hline Gestational age-adjusted birth weight (z-score) & 399 & $-0.25 \pm 0.88$ & -0.27 & $-0.91,0.37$ \\
\hline Gestational age (d) & 399 & $281 \pm 12$ & 281 & 272,288 \\
\hline Birth length $(\mathrm{cm})$ & 398 & $51.2 \pm 2.5$ & 50.8 & $49.5,52.7$ \\
\hline Head circumference (mm) & 397 & $339 \pm 14$ & 343 & 330,349 \\
\hline \multicolumn{5}{|l|}{ Five-year growth outcomes } \\
\hline Sitting height $(\mathrm{cm})$ & 399 & $60.4 \pm 2.8$ & 60.3 & $58.7,62.3$ \\
\hline Standing height $(\mathrm{cm})$ & 397 & $110 \pm 4$ & 110 & 107,113 \\
\hline Sex- and race-standardized height (z-score) & 398 & $49.7 \pm 9.7$ & 50 & 43,56 \\
\hline Sex- and race-standardized weight (z-score) & 399 & $49.2 \pm 9.2$ & 48 & 43,54 \\
\hline Head circumference (mm) & 389 & $510 \pm 15$ & 508 & 498,521 \\
\hline Biacromial distance (mm) & 389 & $245 \pm 16$ & 246 & 236,255 \\
\hline Chest breadth (mm) & 389 & $177 \pm 9.0$ & 176 & 170,183 \\
\hline Chest depth (mm) & 389 & $135 \pm 10.9$ & 134 & 128,142 \\
\hline Bi-iliac distance (mm) & 389 & $174 \pm 10$ & 174 & 167,180 \\
\hline
\end{tabular}

SD indicates standard deviation. 


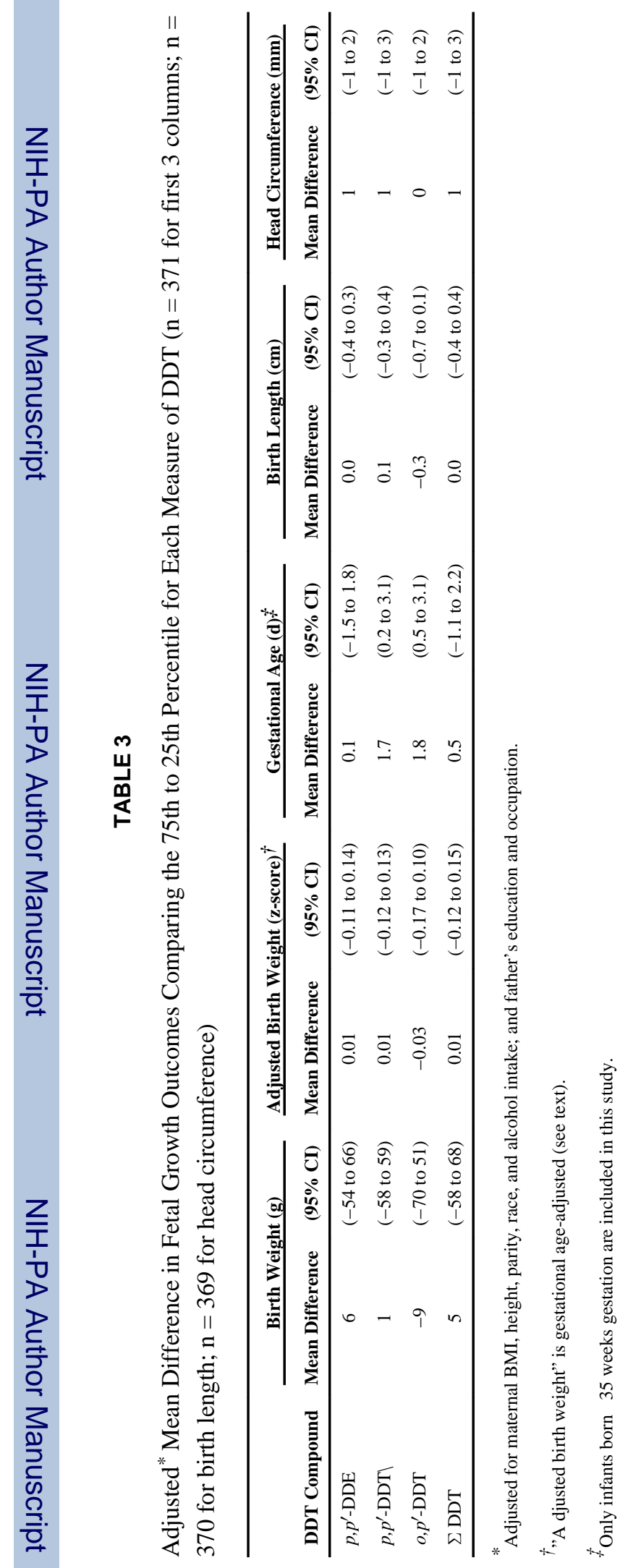




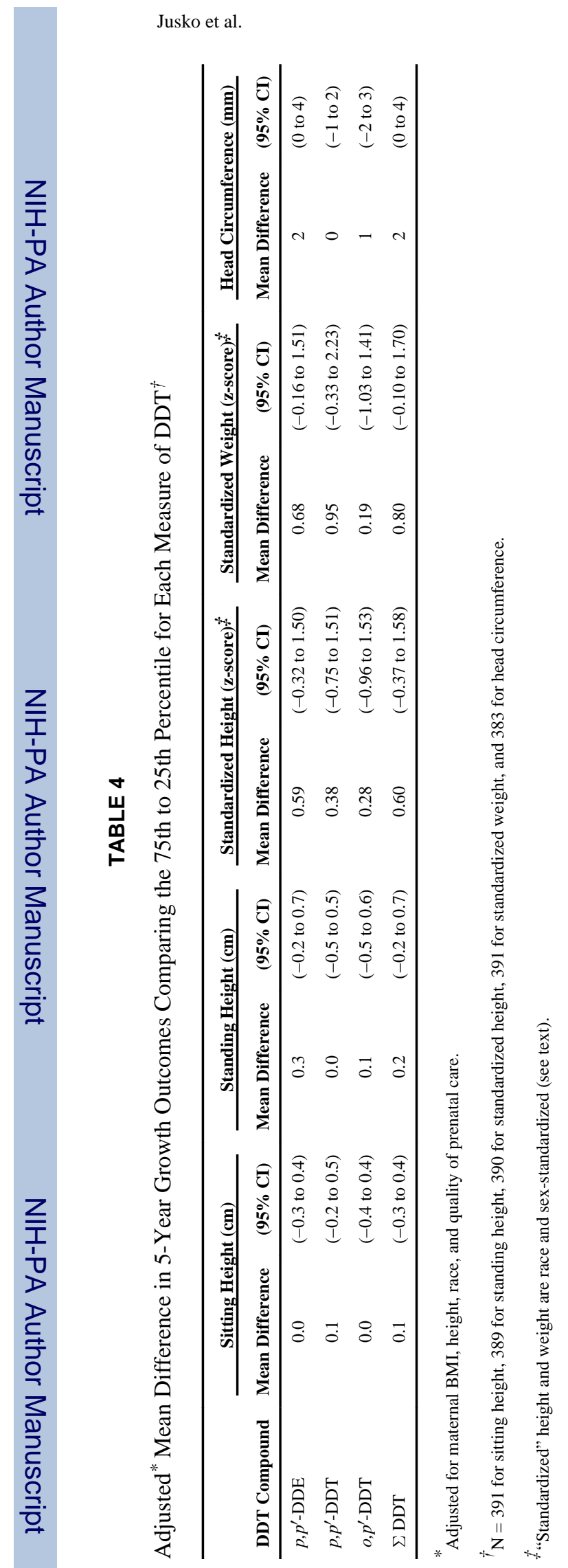

Epidemiology. Author manuscript; available in PMC 2014 June 03. 